\author{
Sandra R. von Schulthess \\ Claude Kaufmann \\ Lucas M. Bachmann \\ Ahmet Yanar \\ Michael A. Thiel
}

\section{Ocular pulse amplitude after trabeculectomy}

Received: 6 February 2005

Revised: 2 June 2005

Accepted: 4 June 2005

Published online: 23 July 2005

(C) Springer-Verlag 2005
This study was presented at the annual scientific meeting of the Swiss Society of Ophthalmology on 17 January 2005.

S. R. von Schulthess - C. Kaufmann .

A. Yanar · M. A. Thiel $(\square)$

Department of Ophthalmology,

University of Zurich,

CH-8091 Zurich, Switzerland

e-mail: michael.thiel@usz.ch

Tel.: +41-1-442558793

Fax: +41-1-442554472

\section{M. Bachmann}

Horten Centre, University of Zurich, Zurich, Switzerland
Abstract Background: The ocular pulse amplitude (OPA) is the difference between the minimum and maximum values of the pulsatile intraocular pressure (IOP) wave contour. The OPA depends on ocular perfusion and IOP, which are both affected by a trabeculectomy (TE). The aim of this study was to investigate how the OPA changes after TE and whether an early change in OPA can be used as a prognostic marker for a successful long-term outcome.

Methods: Fourteen consecutive patients (26-84 years old) with medically uncontrolled primary open-angle or pseudoexfoliation glaucoma were included in the study. IOP and OPA were measured with a dynamic contour tonometer before and after TE on days $-1,+1,+7,+14,+21,+28,+42$, $+56,+70$, and +84 . The OPA of the contralateral eye was used to control for variations in systemic haemodynamics. TE was regarded as successful if a persistent drop in IOP of at least $20 \%$ without the use of IOPlowering treatment was achieved. Data were analysed using receiver operating characteristic curves, Kaplan-Meier survival curves and Mann-Whitney two-sample analysis. Results: Five out of 14 TEs had an entirely successful outcome. The other 9 patients required additional interventions such as suturolysis, needling of subconjunctival scar tissue and antimetabolite injections during the 3-month period after the TE. On the first day after surgery, OPA decreased in 12 patients and increased in 2 patients compared with the preoperative measurements. In the 5 patients with a successful long-term outcome, OPA dropped by $3.38 \pm$ $1.79 \mathrm{mmHg}($ mean $\pm \mathrm{SE})$, whereas the initial OPA drop in those cases that required additional interventions was $0.62+/-1.81 \mathrm{mmHg}$ only $(p<0.01)$. IOP dropped by $13.10 \pm 2.14 \mathrm{mmHg}$ in the successful group and by $5.84 \pm$ $2.51 \mathrm{mmHg}$ in the unsuccessful group $(p=0.19)$. Kaplan-Meier estimates of survival showed that patients with an initial OPA drop of more than $2.0 \mathrm{mmHg}$ had a significantly better chance of an entirely uncomplicated 3-month outcome after TE than patients with an initial OPA drop of less than $2.0 \mathrm{mmHg}(\log$ rank $p<0.01)$. Conclusion: This pilot study indicates that an early drop in OPA of more than $2.0 \mathrm{mmHg}$ after TE may be a good prognostic parameter for successful long-term control of IOP.

Keywords Trabeculectomy - Ocular pulse amplitude - Dynamic contour tonometry 


\section{Introduction}

Standardised evaluation of the bleb morphology after trabeculectomy (TE) has proven to be useful in identifying cases of threatened bleb failure [13]. The management of such patients requires intensive postsurgical follow-up. Subsequent interventions such as suturolysis, subconjunctival antimetabolite injections or bleb-needling procedures are needed for long-term control of intraocular pressure (IOP) if hypotensive medication is to be avoided. However, failure of an individual bleb remains difficult to predict, and additional clinical parameters, especially in the very early postoperative period, would be helpful in tailoring the intensity of the postoperative follow-up protocols to individual needs. We hypothesised that the ocular pulse amplitude (OPA), being the difference between the minimum and maximum values of the ocular pulse wave contour, represents such a parameter. The bulbus resembles a pressurised shell that is subjected to pulsatile expansion generated by the rhythmic filling of intraocular vessels. The corresponding fluctuation of the ocular volume leads to an oscillating variation in IOP that is counterbalanced by the outer shell's resistance to distension [17]. These oscillations are mirrored in the ocular pulse wave (Fig. 1) that can be recorded by continuously measuring tonometers such as the dynamic contour tonometer (Pascal, Swiss Microtechnology, Port, Switzerland) [10]. A successful TE wound creates an additional outflow facility for the aqueous that may compensate in part for the increase in ocular volume during the cardiac systole. Hence, a dampening of the OPA may be expected postoperatively.

The aim of this study was to investigate how the OPA changes after TE and whether an initial drop in OPA has a better predictive value than an initial drop in IOP for the long-term success of trabeculectomy.

\section{Methods}

Fourteen consecutive patients with primary open-angle glaucoma (POAG; $n=7$ ) or pseudoexfoliation glaucoma (PEX; $n=7)$ whose IOP was not adequately controlled by medical therapy and who were undergoing TE for the first time were prospectively included in the study. The patients' characteristics are given in Table 1. After the patients' informed consent had been obtained the IOP and the OPA were measured by dynamic contour tonometry (DCT) on days $-1,+1,+7,+14,+21,+28,+42,+56,+70$ and +84 with respect to the day of surgery. The preoperative assessment included refraction, visual acuity, a detailed slit-lamp examination and fundus examination. Preoperative measurements were taken while the patients were still under medical antihypertensive therapy. There was no wash-out period before surgery. For each time point the mean of four DCT measurements in the sitting position was used to assess both IOP and OPA. To compensate for diurnal OPA fluctuations as a result of fluctuating heart rate and blood pressure, the changes in OPA of the contralateral control eye were deducted from the changes found in the operated eye. All patients were operated on by the same two surgeons performing a standard TE with a fornix-based conjunctival flap, a $3 \times 5-\mathrm{mm}$ scleral flap, a single-punch TE and adjunctive use of mitomycin $\mathrm{C}(0.025 \%$ for $2 \mathrm{~min})$. Postoperative treatment consisted of intensive topical antiinflammatory treatment $(0.1 \%$ dexamethasone phosphate drops every $2 \mathrm{~h}$ and ointment at night). The outcome after
Fig. 1 Ocular pulse wave contour as measured by dynamic contour tonometry. The device displays the diastolic readings as intraocular pressure (IOP), and the difference between the means of systolic and diastolic readings is furnished as ocular pulse amplitude (OPA)

\section{Ocular Pulse Amplitude (OPA)}

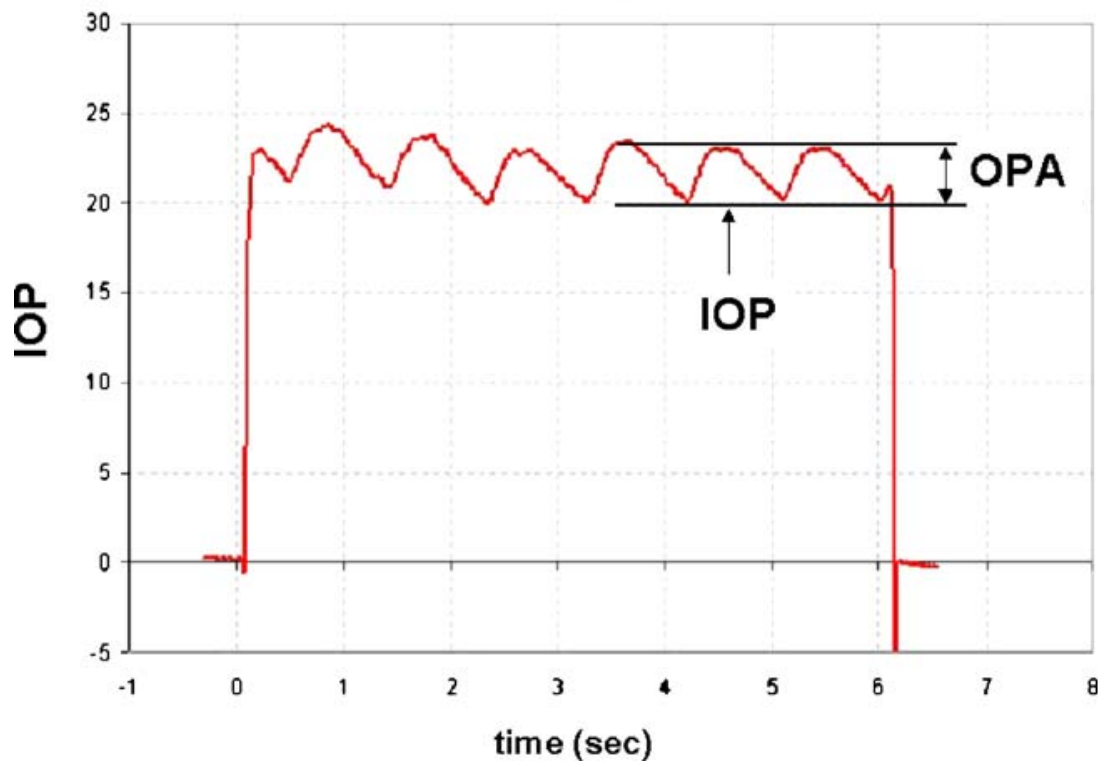




\begin{tabular}{|c|c|c|c|c|c|c|c|}
\hline $\begin{array}{l}\text { Table } 1 \text { Characteristics of the } \\
\text { study group }\end{array}$ & $\begin{array}{l}\text { Patient } \\
\text { number }\end{array}$ & Gender & $\begin{array}{l}\text { Age } \\
\text { (years) }\end{array}$ & $\begin{array}{l}\text { Type of } \\
\text { glaucoma }\end{array}$ & $\begin{array}{l}\text { Preoperative IOP } \\
(\mathrm{mmHg})\end{array}$ & $\begin{array}{l}\text { Treatment before } \\
\text { surgery }\end{array}$ & $\begin{array}{l}\text { Outcome } \\
\text { (1=failure) }\end{array}$ \\
\hline & 1 & $\mathrm{~F}$ & 70 & PEX & 21.5 & Xalacom & 1 \\
\hline & 2 & $\mathrm{~F}$ & 80 & PEX & 33 & Cosopt, Xalatan & 1 \\
\hline & 3 & $\mathrm{~F}$ & 74 & PEX & 16.8 & Xalatan & 0 \\
\hline & 4 & M & 60 & POAG & 21.3 & Vistagan & 0 \\
\hline & 5 & $\mathrm{~F}$ & 84 & POAG & 21.3 & Xalatan, Azopt & 1 \\
\hline & 6 & $\mathrm{~F}$ & 82 & PEX & 16.7 & Lumigan & 1 \\
\hline & 7 & M & 67 & PEX & 14.5 & Xalacom, Alphagan & 0 \\
\hline & 8 & $\mathrm{~F}$ & 69 & POAG & 18.6 & Travatan & 1 \\
\hline & 9 & $\mathrm{~F}$ & 61 & PEX & 22.8 & Cosopt, Xalacom & 0 \\
\hline & 10 & $\mathrm{~F}$ & 74 & POAG & 25.9 & Xalacom & 1 \\
\hline & 11 & M & 38 & POAG & 22.1 & $\begin{array}{l}\text { Xalatan, Alphagan, } \\
\text { Cosopt }\end{array}$ & 0 \\
\hline & 12 & $\mathrm{~F}$ & 58 & PEX & 22.7 & $\begin{array}{l}\text { Travatan, Cosopt, } \\
\text { Alphagan }\end{array}$ & 1 \\
\hline & 13 & $\mathrm{M}$ & 26 & POAG & 31.2 & $\begin{array}{l}\text { Timoptic, Alphagan, } \\
\text { Xalatan }\end{array}$ & 1 \\
\hline & 14 & $\mathrm{M}$ & 73 & POAG & 29.4 & $\begin{array}{l}\text { Cosopt, Alphagan, } \\
\text { Xalatan }\end{array}$ & 1 \\
\hline
\end{tabular}

TE was regarded as successful if a postoperative drop in IOP of at least $20 \%$ at any time point was achieved without the use of hypotensive treatment, laser suturolysis or needling revisions [13].

Two different analytical approaches were performed: To calculate the optimal cut-off value for the OPA and IOP drop we plotted receiver operating characteristic (ROC) curves. At these cut-off values we calculated the diagnostic odds ratios. We then compared the areas under the ROC curve of the two tests using the method proposed by Hanley and McNeil to assess the predictive value of an initial drop in OPA or IOP after surgery [7].

Second, we assessed the likelihood of an uncomplicated outcome after trabeculectomy in a survival analysis where we compared patients who showed a decrease in OPA of at least $2.0 \mathrm{mmHg}$ on the first postoperative day with those experiencing a lower drop. Kaplan-Meier survival curves were compared using the log rank test. Differences in mean values between groups were tested using the Mann-Whitney two-sample statistic. A $p$ value of $<0.05$ was considered significant. Data are expressed as mean \pm standard deviation.

To estimate the reliability of the IOP and OPA measurements obtained by DCT we performed two analyses of variance (ANOVAs) for the preoperative and first postoperative measurements. For these analyses four measurements for each patient were available at the two time points. We used patient as the random factor, IOP and OPA as the dependent variables. Based on the variance components, we calculated the intraclass correlation coefficients (ICCs) using the patient variance in the numerator and the sum of residual and patient variance in the denominator. From the variance component procedure, we used the var- iance component of the residual error as the intraobserver variability.

\section{Results}

Five out of the 14 patients had an entirely successful outcome after TE with an IOP drop of at least $20 \%$ at any time point after surgery. The other nine patients cases required additional interventions such as suturolysis $(n=4)$, needling of subconjunctival scar tissue $(n=6)$ and antimetabolite injections $(n=6)$ during the 3-month follow-up period. Before surgery, baseline IOP and OPA did not differ between the groups that subsequently experienced successful or unsuccessful TE (IOP: $19.50 \pm 3.64 \mathrm{mmHg}$ vs $24.47 \pm$ $5.71 \mathrm{mmHg}, p=0.10$; OPA: $3.74 \pm 1.15 \mathrm{mmHg}$ vs $4.00 \pm$ $1.91 \mathrm{mmHg}, p=0.75$ ).

On the first day after surgery, OPA had dropped by $3.38 \pm$ $1.79 \mathrm{mmHg}$ in patients with a successful long-term outcome, whereas the decrease in patients that eventually required additional interventions was only $0.62 \pm 1.81 \mathrm{mmHg}$ $(p<0.01)$. The latter group comprises the only three patients who experienced an increase in OPA instead of a decrease.

The IOP decreased on the first postoperative day in 13 out of 14 patients, the exception being one of the members of the failure group. The initial drop in IOP was $13.10 \pm$ $2.14 \mathrm{mmHg}$ in cases with successful outcome and 5.84 \pm $2.51 \mathrm{mmHg}$ in cases with unsuccessful outcome respectively (Fig. 2, $p=0.19$ ). In the patients with successful TE the OPA decreased by $0.30 \pm 0.25 \mathrm{mmHg}$ per $1-\mathrm{mmHg}$ drop in IOP, while the OPA in patients that required subsequent interventions initially dropped by only $0.004 \pm$ $0.49 \mathrm{mmHg}$ per $1-\mathrm{mmHg}$ drop in IOP $(p=0.09)$. The ROC 
Fig. 2 Mean IOP reduction after TE. The outcome after TE was regarded as successful if a postoperative drop in IOP of at least $20 \%$ was achieved at any time point without the use of any hypotensive treatment, laser suturolysis or bleb-needling procedures
Kaplan-Meier survival estimates

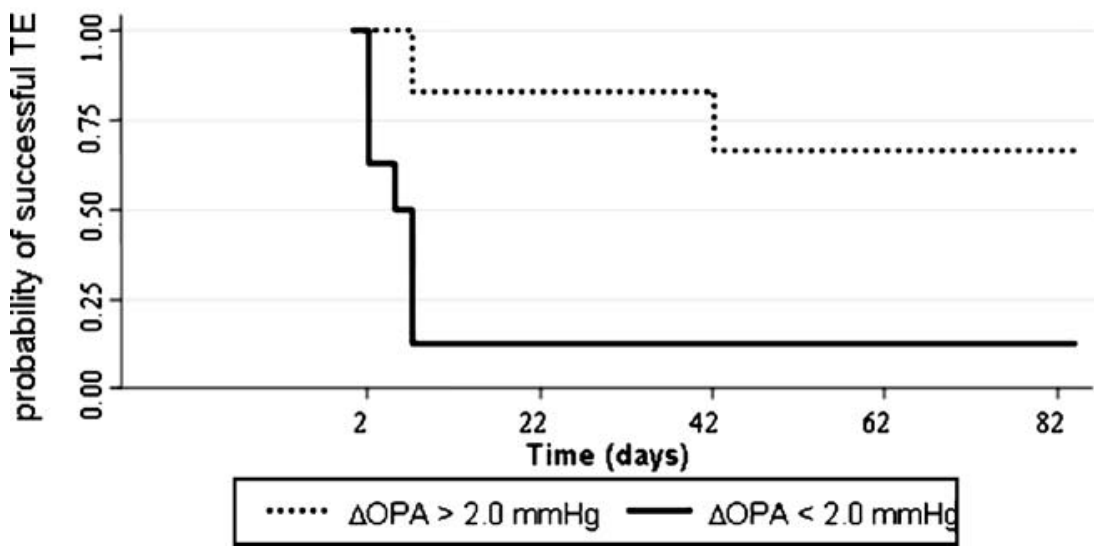

curves indicated that a decrease in OPA of at least 2.0 $\mathrm{mmHg}$ and a drop in the IOP of more than $9 \mathrm{mmHg}$ by the time of measurement on the first postoperative day were most predictive for treatment success. The area under the ROC curve for an OPA drop was 0.91 (95\% confidence interval $0.70-1.00)$ and $0.78(95 \% \mathrm{CI} 0.50-1.00)$ for an IOP drop, respectively $(\mathrm{p}=0.22)$. The corresponding odds ratios were $14(95 \% \mathrm{CI} 1.0-208)$ and $8(0.5-107)$, indicating that an OPA drop was more accurate than an IOP drop as predictor of a successful outcome after TE.

Kaplan-Meier estimates of survival showed that patients with an initial drop in OPA of more than $2.0 \mathrm{mmHg}$ had a significantly better chance of experiencing an entirely uncomplicated 3-month outcome, while patients with an initial decrease in OPA of less than $2.0 \mathrm{mmHg}$ had a high probability of failure requiring additional interventions (log rank $p=0.01$, Fig. 3).

Assessment of the reliability of the IOP and OPA measurements before and after TE showed preoperative and postoperative ICCs for IOP of 0.85 and 0.95 . The corresponding ICCs for OPA were 0.5 and 0.79 respectively, indicating a high reliability for IOP measurements and the

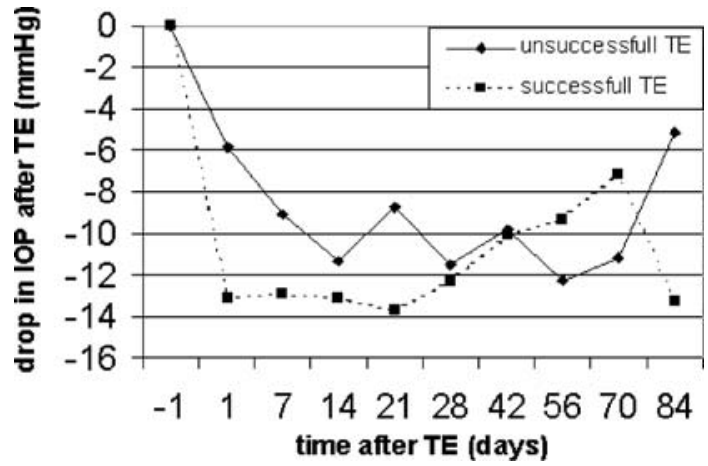

Fig. 3 Kaplan-Meier survival analysis of TE with an OPA drop on the first postoperative day of more or less than $2.0 \mathrm{mmHg}$ compared with the presurgical baseline OPA. Patients with a OPA drop of more than $2.0 \mathrm{mmHg}$ have an higher probability of a successful outcome after TE $(p=0.001)$ postoperative OPA measurements. The preoperative intraobserver variability for the OPA was $1.6 \mathrm{mmHg}$, whereas the variability of the first postoperative measurements was $0.7 \mathrm{mmHg}$.

\section{Discussion}

This pilot study documents that the OPA goes down after TE and that an OPA drop of more than $2.0 \mathrm{mmHg}$ on the first day after TE may be a prognostic indicator of a favourable outcome without the need for additional interventions.

Reports on OPA after TE are sparse: James investigated the effect of TE on pulsatile ocular blood flow (POBF) using a Langham OBF system, recording the OPA in 17 patients before and 3 and 6 months after TE [8]. In the vertical position the drop in OPA $(0.4 \mathrm{mmHg})$ became significant only 6 months after surgery. The POBF, which is based on a measurement of the OPA and recalculated into a POBF value by the Langham OBF system, was found to increase after TE. Similarly, Poinoosawmy et al. found an increased POBF 2 weeks after TE using the same device but did not report the OPA [15]. Both studies measured OPA to investigate the long-term change in OBF, which is recognised as an important haemodynamic factor for the pathogenesis of glaucomatous optic neuropathy (for review see [3]). However, calculations of the POBF that are based on the OPA may become unreliable, as the calculations depend on the rigidity of the eyeball, which is altered by the TE [20]. Our study differs from the studies cited above in that we measured the OPA on the first day after TE by DCT to investigate the role of the OPA as an prognostic parameter for successful TE.

At this stage, we can only speculate about the underlying mechanisms responsible for the more pronounced OPA decrease in patients with successful TE than in those with less successful TE requiring further interventions. An overall reduction in scleral rigidity following successful filtering operation has been suggested as a potential cause of fall 
in OPA [20]. In addition to the above-mentioned change in aqueous humour dynamics, the volume-pressure relation of the human eye provides another explanation: it is known for several decades that the ocular shell's resistance to distension varies with the general IOP level and that the OPA consecutively decreases as the IOP level is lowered [19]. In this study, however, patients with an entirely successful outcome after TE showed a higher drop in OPA per $1-\mathrm{mmHg}$ drop in IOP than patients with an unsuccessful outcome. Therefore we might speculate that the more pronounced drop in OPA found in patients with an entirely successful outcome after TE reflects a lower outflow resistance in these cases.

Alternatively, the less favourable outcome of the TE in cases with a higher OPA after surgery may be caused by biological differences in wound healing. The higher OPA reflects a higher amplitude in the pulsatile ocular wall tension, which may result in a different activation or modulation of the fibroblasts involved in the ocular wound healing after TE $[1,5]$. However, this aspect was beyond the scope of this pilot study.

It is important to note that parameters such as axial length $[9,14]$ and heart rate $[6,18]$ have not been considered in this study although an impact on the OPA has been described. Reduction of axial length, as observed in successful trabeculectomies [4], may result in an increased OPA [14] and would therefore rather have hidden the OPA-lowering effect found in this study in patients with successful TE. To control for individual fluctuations of cardiovascular parameters such as heart rate or blood pressure, the changes in the OPA of the contralateral eye were used as controls. Additional confounding effects may have arisen from the hypotensive medication itself [16], as there was no wash-out phase preoperatively. It has been suggested that the effect of antiglaucoma medication on ocular haemodynamics may also be due to a vascular effect unrelated to the decrease in IOP [2]. However, in our study neither patients with a successful TE nor those with an unsuccessful TE received any pressure-lowering drugs to the operated eye during the 3-month study period after TE.

Direct comparisons between studies on ocular pulse waves must be made cautiously, as the accuracy and reliability of the measuring tonometer are crucial [12]. DCT has been shown to compare favourably to Goldmann applanation tonometry with respect to inter- and intraobserver variability $(0.65 \mathrm{mmHg}$ and $0.44 \mathrm{mmHg}$ respectively) and independence from corneal curvature and thickness [10]. Probably even more important for the evaluation of ocular haemodynamics is the finding that DCT reproduces the manometrically controlled IOP level with high fidelity [11].

The low probability of a successful outcome after TE in patients with a drop in OPA of less than $2.0 \mathrm{mmHg}$ on the first day after surgery is an important finding. The OPA represents a parameter which can be easily measured in clinical routine and which might prove useful at an early stage after TE in those patients who are in need of frequent follow-up appointments and possibly additional interventions. However, this was a pilot study in a small number of patients, possibly confounded by different types of glaucoma and other yet unknown factors that could influence OPA measurements. Until the findings of this pilot study are confirmed by larger, well-controlled studies, patients should be monitored closely after TE irrespective of an early drop in OPA.

\section{References}

1. Ajubi NE, Klein-Nulend J, Nijweide PJ, Vrijheid-Lammers T, Alblas MJ, Burger EH (1996) Pulsating fluid flow increases prostaglandin production by cultured chicken osteocytes - a cytoskeleton-dependent process. Biochem Biophys Res Commun 225:62-68

2. Cantor LB (2001) The effect of trabeculectomy on ocular hemodynamics. Trans Am Ophthalmol Soc 99:241-252

3. Flammer J, Orgul S, Costa VP, Orzalesi N, Krieglstein GK, Serra LM, Renard JP, Stefansson E (2002) The impact of ocular blood flow in glaucoma. Prog Retin Eye Res 21:359-393
4. Francis BA, Wang M, Lei H, Du LT, Minckler DS, Green RL, Roland C (2005) Changes in axial length following trabeculectomy and glaucoma drainage device surgery. Br J Ophthalmol 89:17-20

5. Fujikura H, Seko Y, Tokoro T, Mochizuki M, Shimokawa H (2002) Involvement of mechanical stretch in the gelatinolytic activity of the fibrous sclera of chicks, in vitro. Jpn J Ophthalmol 46:24-30

6. Gekkieva M, Orgul S, Gherghel D, Gugleta K, Prunte C, Flammer J (2001) The influence of sex difference in measurements with the Langham Ocular Blood Flow System. Jpn J Ophthalmol 45:528-532
7. Hanley JA, McNeil BJ (1983) A method of comparing the areas under receiver operating characteristic curves derived from the same cases. Radiology 148:839-843

8. James CB (1994) Effect of trabeculectomy on pulsatile ocular blood flow. Br J Ophthalmol 78:818-822

9. James CB, Trew DR, Clark K, Smith SE (1991) Factors influencing the ocular pulse-axial length. Graefes Arch Clin Exp Ophthalmol 229:341-344

10. Kaufmann C, Bachmann LM, Thiel MA (2004) Comparison of dynamic contour tonometry with Goldmann applanation tonometry. Invest Ophthalmol Vis Sci 45:3118-3121 
11. Kniestedt C, Nee M, Stamper RL (2004) Dynamic contour tonometry: a comparative study on human cadaver eyes. Arch Ophthalmol 122:1287-1293

12. Krakau CET (1992) Calculation of the pulsatile ocular blood flow. Invest Ophthalmol Vis Sci 33:2754-2756

13. Marquardt D, Lieb WE, Grehn F (2004) Intensified postoperative care versus conventional follow-up: a retrospective long-term analysis of 177 trabeculectomies. Graefes Arch Clin Exp Ophthalmol 242:106-113
14. Perkins ES (1981) The ocular pulse. Curr Eye Res 1:19-23

15. Poinoosawmy D, Indar A, Bunce C, Garway-Heath DF, Hitchings RA (2002) Effect of treatment by medicine or surgery on intraocular pressure and pulsatile ocular blood flow in normalpressure glaucoma. Graefes Arch Clin Exp Ophthalmol 240:721-726

16. Schmidt KG, Dick B, von Ruckmann A, Pillunat LE (1997) Ocular pulse amplitude and local carbonic anhydrase inhibition. Ophthalmologe 94:659-664

17. Silver DM, Geyer O (2000) Pressurevolume relation for the living human eye. Curr Eye Res 20:115-120
18. Trew DR, James CB, Thomas SH, Sutton R, Smith SE (1991) Factors influencing the ocular pulse-the heart rate. Graefes Arch Clin Exp Ophthalmol 229:553-556

19. Wegner W (1930) Neue Ergebnisse ueber die pulsatorischen Schwankungen des menschlichen Bulbus und seiner Huellen. Arch Augenheilkd 102:1-32

20. Yang YC, Hulbert MF (1995) Effect of trabeculectomy on pulsatile ocular blood flow. Br J Ophthalmol 79:507508 